\title{
Decay of Correlations for Infinite Range Interactions in Unbounded Spin Systems`
}

\author{
Camillo Cammarota \\ Istituto di Matematica, Università di Roma, C.N.R. (G.N.F.M.), I-00185 Roma, Italy
}

\begin{abstract}
In unbounded spin systems at high temperature with two-body potential we prove, using the associated polymer model, that the two-point truncated correlation function decays exponentially (respectively with a power law) if the potential decays exponentially (respectively with a power law). We also give a new proof of the convergence of the Mayer series for the general polymer model.
\end{abstract}

\section{Definitions and Results}

In the finite subset $\Lambda$ of $\mathbb{Z}^{d}$ we consider the collection of random variables $S_{\Lambda}$ $=\left\{S_{x} \in \mathbb{R}^{v}, x \in \Lambda\right\}$ distributed with the Gibbs probability measure, i.e.,

$$
Z_{\Lambda}^{-1} e^{-\beta \sum_{\substack{X \in A \\|X| \geqq 2}} \Phi_{X}\left(S_{X}\right)} W_{A}\left(d S_{A}\right),
$$

where $\Phi$ is a given many-body potential,

$$
\begin{aligned}
& W_{\Lambda}\left(d S_{\Lambda}\right)=\prod_{x \in \Lambda} W_{x}\left(d S_{x}\right), \\
& W_{x}\left(d S_{x}\right)=\left(\int \mu_{x}\left(d S_{x}\right) \exp -\beta \Phi_{x}\left(S_{x}\right)\right)^{-1}\left(\exp -\beta \Phi_{x}\left(S_{x}\right)\right) \mu_{x}\left(d S_{x}\right),
\end{aligned}
$$

where $\mu_{x}$ is the a priori single spin distribution and $\beta$ is the inverse temperature, $Z_{A}$ is the partition function and $|X|$ is the number of points of $X$.

The finite volume correlation functions are

$$
\varrho_{\Lambda}\left(S_{X}\right)=Z_{\Lambda}^{-1} \int W_{\Lambda \backslash X}\left(d S_{\Lambda \backslash X}\right) \exp -\beta \sum_{\substack{X \subset A \\|X| \geqq 2}} \Phi_{X}\left(S_{X}\right) .
$$

Our first result is the following theorem :

Theorem 1. Let $\Phi$ be a two-body potential such that

$$
\left|\Phi_{x y}\left(S_{x} S_{y}\right)\right| \leqq e^{-\delta(x, y)} J(x, y) v_{x}\left(S_{x}\right) v_{y}\left(S_{y}\right),
$$

* Supported by C.N.R. (G.N.F.M.) 
where $\delta$ is a metric on $\mathbb{Z}^{d}$ such that

$$
\sup _{x \in \mathbb{Z}^{d}} \sum_{y \in \mathbb{Z}^{d} \backslash\{x\}} e^{-\frac{1}{2} \delta(x, y)}=D,
$$

$J(x, y)$ satisfies

$$
\sup _{x \in \mathbb{Z}^{d}} \sum_{y \in \mathbb{Z}^{d} \backslash\{x\}} J(x, y)^{1 / 3}=J
$$

and $v_{x}$ is such that

$$
\begin{gathered}
\sup _{x \in \mathbb{Z}^{d}} \int W_{x}\left(d S_{x}\right) \exp \frac{1}{2} J v_{x}\left(S_{x}\right)^{2}=I(\beta), \\
I(\beta)=O(1), \quad \beta \rightarrow 0 .
\end{gathered}
$$

Then there are two functions, $I_{3}(\beta)$ and $I_{11}(\beta)$, both $O(\sqrt{\beta}), \beta \rightarrow 0$ such that, if

$$
\begin{aligned}
I(\beta) J & \sqrt{\beta} \exp D I_{3}(\beta)<1, \\
& \quad \sup _{\{x, y\}} J(x, y)^{2 / 3}<1, \\
& \frac{I(\beta) J^{1 / 2} \beta^{1 / 4}}{1-J \beta^{1 / 2}}<\frac{1}{2 e+1},
\end{aligned}
$$

we have for each $\Lambda$

$$
\left|\varrho_{\Lambda}\left(S_{x} S_{y}\right)-\varrho_{\Lambda}\left(S_{x}\right) \varrho_{\Lambda}\left(S_{y}\right)\right| \leqq e^{\frac{1}{2} J v_{x}\left(S_{x}\right)^{2}+\frac{1}{2} J v_{y}\left(S_{y}\right)^{2}} e^{-\frac{1}{2} \delta(x, y)} I_{11}(\beta) .
$$

This theorem eliminates the finite range assumption on the potential present in a similar theorem in [1], leaving essentially unchanged the other hypothesis. The infinite range case has been already considered in $[2,3]$, but for special classes of systems. We refer to [1] also for a discussion on the physical meaning of the main hypothesis (1.7) and for the proof of the existence of the infinite volume correlation functions to which, obviously, in a suitable range of $\beta$ the bound (1.11) applies. For the use of the term $\exp -\delta$ in the potential we refer, for instance, to [4].

The main idea of the proof of Theorem 1 is to use the Mayer expansion for the polymer model associated to our system. Let us recall the polymer model $[1,5]$. A polymer is a finite subset of $\mathbb{Z}^{d}$ and its activity is given by

$$
\begin{gathered}
\zeta(R)=\int W_{R}\left(d S_{R}\right) \zeta\left(S_{R}\right), \\
\zeta\left(S_{R}\right)= \begin{cases}1 & |R|=1 \\
\sum_{K \geqq 1} \frac{1}{K !} \sum_{\left(X_{1}, \ldots, X_{K}\right)}^{*} \prod_{i=1}^{K}\left(e^{-\beta \Phi_{X_{i}}\left(S_{X_{\imath}}\right)}-1\right) & |R|>1,\end{cases}
\end{gathered}
$$

where * means that the sum runs over the $K$-sequences of subsets of $R$ with $\left|X_{i}\right| \geqq 2$, $X_{i} \neq X_{j}, U X_{i}=R$ and, denoted by $g\left(X_{1}, \ldots, X_{K}\right)$ the graph on $\{1, \ldots, K\}$ that has a line $\{i, j\}$ if and only if $X_{i} \cap X_{j} \neq \emptyset$, the graph $g\left(X_{1}, \ldots, X_{K}\right)$ is connected. In force of this definition

$$
Z_{\Lambda}=\sum_{n \geqq 1} \sum_{\left\{R_{1}, \ldots, R_{n}\right\} \in \pi(\Lambda)} \zeta\left(R_{1}\right) \ldots \zeta\left(R_{n}\right)
$$


where $\pi(\Lambda)$ is the set of the partitions of $\Lambda$. The correlation functions of the polymer model are

$$
\bar{\varrho}_{\Lambda}(X)=Z_{\Lambda}^{-1} \sum_{n \geqq 1} \sum_{\left\{R_{1}, \ldots, R_{n}\right\} \in \pi(\Lambda \backslash X)} \zeta\left(R_{1}\right) \ldots \zeta\left(R_{n}\right)=Z_{\Lambda}^{-1} Z_{\Lambda \backslash X}
$$

and, using them, the correlation functions of the system can be conveniently expressed

$$
\varrho_{\Lambda}\left(S_{X}\right)=\sum_{Y \subset \Lambda \backslash X} \bar{\varrho}_{\Lambda}(X \cup Y) \int W_{Y}\left(d S_{Y}\right) F_{S_{X}}\left(S_{Y}\right),
$$

where

$$
F_{S_{X}}\left(S_{Y}\right)=\sum_{n \geqq 1} \sum_{\substack{\left\{R_{1}, \ldots, R_{n}\right\} \in \pi(X \cup Y) \\ R_{i} \cap X \neq \emptyset}} \zeta\left(S_{R_{1}}\right) \ldots \zeta\left(S_{R_{n}}\right) .
$$

The Mayer series for the general polymer model is given by the following theorem in which appears the combinatorial function $\varphi^{T}$ (truncated function) that we define on $\bigcup_{n \geqq 1} \mathscr{R}^{n}$, where $\mathscr{R}$ is the set of the polymers $R$ with $|R| \geqq 2$ :

$$
\varphi^{T}\left(R_{1}, \ldots, R_{n}\right)= \begin{cases}1 & n=1 \\ \sum_{g \in C_{n}} \prod_{\{i, j\} \in g}\left(\chi\left(R_{i}, R_{j}\right)-1\right) & n>1\end{cases}
$$

where $C_{n}$ is the set of the connected graphs on $\{1, \ldots, n\}$ and

$$
\chi\left(R_{i}, R_{j}\right)= \begin{cases}0 & R_{i} \cap R_{j} \neq \emptyset \\ 1 & R_{i} \cap R_{j}=\emptyset .\end{cases}
$$

Theorem 2. If $\zeta$ satisfies, for each integer $K \geqq 2$,

$$
\sup _{x \in \mathbb{Z}^{d}} \sum_{\substack{x \in R \in \mathscr{R} \\|R|=K}}|\zeta(\mathrm{R})| \leqq \varepsilon^{K}
$$

and

$$
\frac{\varepsilon}{1-\varepsilon}<\frac{1}{2 e}
$$

then

$$
\begin{aligned}
& \sum_{n=1}^{\infty} \frac{1}{n !} \sum_{\substack{\left(R_{1}, \ldots, R_{n}\right) \in \mathscr{R}_{n} \\
\exists R_{i}=R}}\left|\varphi^{T}\left(R_{1}, \ldots, R_{n}\right) \zeta\left(R_{1}\right) \ldots \zeta\left(R_{n}\right)\right| \\
& \quad \leqq|\zeta(R)|\left(1+|R| e^{|R|} \frac{1}{2} \ln \left(1-2 e \frac{\varepsilon}{1-\varepsilon}\right)^{-1}\right)
\end{aligned}
$$

and the exponentiation formula holds, i.e.,

$$
Z_{\Lambda}=\exp \sum_{n=1}^{\infty} \frac{1}{n !} \sum_{\substack{\left(R_{1}, \ldots, R_{n}\right) \in \mathscr{R}^{n} \\ R_{i} \subset A}} \varphi^{T}\left(R_{1}, \ldots, R_{n}\right) \zeta\left(R_{1}\right) \ldots \zeta\left(R_{n}\right) .
$$


The classical method of proof of this theorem goes back to [6] and uses the "algebraic method" and integral equations of Kirkwood-Salsburg type [7]. (In $[8,9]$ the theorem is explicitly stated for the polymer model.) We present here a new proof of this theorem that shows the peculiar combinatorial aspects of the Mayer series of polymers, making clearer the reasons of its convergence. Nonstandard proofs of the convergence of the Mayer series for continuous systems are already known (see, for instance $[10,11]$ ). The proof of Theorem 2 is in Sect. 3. We use it to prove Theorem 1 in the next section.

\section{Proof of Theorem 1}

We get from (1.15), introducing the shortened notation

$$
\begin{aligned}
F_{S_{X}}(T)= & \int W_{T}\left(d S_{T}\right) F_{S_{X}}\left(S_{T}\right) \\
\varrho_{\Lambda}\left(S_{x} S_{y}\right)-\varrho_{\Lambda}\left(S_{x}\right) \varrho_{\Lambda}\left(S_{y}\right)= & \sum_{T \subset \Lambda \backslash\{x, y\}} \varrho_{\Lambda}(x y T) F_{S_{x} S_{y}}(T) \\
& -\sum_{T_{1} \subset \Lambda \backslash\{x\}} \bar{\varrho}_{\Lambda}\left(x T_{1}\right) F_{S_{x}}\left(T_{1}\right) \sum_{T_{2} \subset \Lambda \backslash\{y\}} \bar{\varrho}_{\Lambda}\left(y T_{2}\right) F_{S_{y}}\left(T_{2}\right) .
\end{aligned}
$$

Equation (1.16) gives, putting

$$
\begin{gathered}
\zeta\left(S_{x} T\right)=\int W_{T}\left(d S_{T}\right) \zeta\left(S_{x} \cup T\right), \\
F_{S_{x}}(T)=\zeta\left(S_{x} T\right), \\
F_{S_{x} S_{y}}(T)=\zeta\left(S_{x} S_{y} T\right)+\sum_{T_{1} \subset T} \zeta\left(S_{x} T_{1}\right) \zeta\left(S_{y} T \backslash T_{1}\right),
\end{gathered}
$$

and so

$$
\begin{aligned}
\varrho_{\Lambda}\left(S_{x} S_{y}\right)-\varrho_{\Lambda}\left(S_{x}\right) \varrho_{\Lambda}\left(S_{y}\right)= & \sum_{T \subset \Lambda \backslash\{x, y\}} \zeta\left(S_{x} S_{y} T\right) \varrho_{\Lambda}(x y T) \\
& +\sum_{T_{1} \subset \Lambda \backslash\{x, y\}} \sum_{\substack{T_{2} \subset A \backslash\{x, y\} \\
T_{2} \cap T_{1}=\emptyset}} \zeta\left(S_{x} T_{1}\right) \zeta\left(S_{y} T_{2}\right) \bar{\varrho}_{\Lambda}\left(x y T_{1} T_{2}\right) \\
& -\sum_{T_{1} \subset \Lambda \backslash\{x\}} \sum_{T_{2} \subset \Lambda \backslash\{y\}} \zeta\left(S_{x} T_{1}\right) \zeta\left(S_{y} T_{2}\right) \bar{\varrho}_{\Lambda}\left(x T_{1}\right) \bar{\varrho}_{\Lambda}\left(y T_{2}\right) .
\end{aligned}
$$

We perform some obvious manipulations quite similar to the ones in [1] and get

$$
\varrho_{\Lambda}\left(S_{x} S_{y}\right)-\varrho_{\Lambda}\left(S_{x}\right) \varrho_{\Lambda}\left(S_{y}\right)=\Sigma_{1}+\ldots+\Sigma_{6}
$$

where

$$
\begin{aligned}
& \Sigma_{1}=\sum_{T \subset \Lambda \backslash\{x, y\}} \zeta\left(S_{x} S_{y} T\right) \bar{\varrho}_{\Lambda}(x y T), \\
& \Sigma_{2}=\sum_{T_{1} \subset \Lambda \backslash\{x, y\}} \sum_{\substack{T_{2} \subset \Lambda \backslash\{x, y\} \\
T_{2} \cap T_{1}=\emptyset}} \zeta\left(S_{x} T_{1}\right) \zeta\left(S_{y} T_{2}\right)\left(\bar{\varrho}_{\Lambda}\left(x y T_{1} T_{2}\right)-\bar{\varrho}\left(x T_{1}\right) \bar{\varrho}_{\Lambda}\left(y T_{2}\right)\right), \\
& \Sigma_{3}=-\sum_{T_{1} \subset \Lambda \backslash\{x, y\}} \sum_{\substack{T_{2} \subset \Lambda \backslash\{x, y\} \\
T_{2} \cap T T_{1} \neq \emptyset}} \zeta\left(S_{x} T_{1}\right) \zeta\left(S_{y} T_{2}\right) \bar{\varrho}_{\Lambda}\left(x T_{1}\right) \bar{\varrho}_{\Lambda}\left(y T_{2}\right),
\end{aligned}
$$




$$
\begin{aligned}
& \Sigma_{4}=-\sum_{y \in T_{1} \subset A \backslash\{x\}} \sum_{T_{2} \subset A \backslash\{x, y\}} \zeta\left(S_{x} T_{1}\right) \zeta\left(S_{y} T_{2}\right) \bar{\varrho}_{\Lambda}\left(x T_{1}\right) \bar{\varrho}_{\Lambda}\left(y T_{2}\right), \\
& \Sigma_{5}=-\sum_{T_{1} \subset A \backslash\{x, y\}} \sum_{x \in T_{2} \subset A \backslash\{y\}} \zeta\left(S_{x} T_{1}\right) \zeta\left(S_{y} T_{2}\right) \bar{\varrho}_{\Lambda}\left(x T_{1}\right) \bar{\varrho}_{\Lambda}\left(y T_{2}\right), \\
& \Sigma_{6}=-\sum_{y \in T_{1} \subset A \mid\{x\}} \sum_{x \in T_{2} \subset A \mid\{y\}} \zeta\left(S_{x} T_{1}\right) \zeta\left(S_{y} T_{2}\right) \bar{\varrho}_{\Lambda}\left(x T_{1}\right) \bar{\varrho}_{\Lambda}\left(y T_{2}\right) .
\end{aligned}
$$

We use the following two lemmas to estimate the terms $\Sigma_{1}, \ldots, \Sigma_{6}$.

Lemma 1. In the hypothesis (1.4), (1.6), (1.7), (1.8), (1.9), we have

$$
\begin{aligned}
& \sup _{x} \sum_{\substack{x \in R \in \mathscr{R} \\
|R|=K}}|\zeta(R)| \leqq I(\beta)^{K}(J \sqrt{\beta})^{K-1}(1-J \sqrt{\beta})^{-1}, \\
& \sup _{\{x, y\}} \sum_{\substack{\{x, y\}<R \in \mathscr{R} \\
|R|=K}}|\zeta(R)| \leqq e^{-\delta(x, y)} I(\beta)^{K}(J \sqrt{\beta})^{K-1}(1-J \sqrt{\beta})^{-1}, \\
& \sup _{\{x, y\}} \sum_{\substack{T \subset \mathbb{Z}^{d} \\
|T|=K}} \int W_{T}\left(d S_{T}\right)\left|\zeta\left(S_{x} S_{y} S_{T}\right)\right| \\
& \quad \leqq e^{\frac{1}{2} J v_{x}\left(S_{x}\right)^{2}+\frac{1}{2} J v_{y}\left(S_{y}\right)^{2}} e^{-\delta(x, y)} I(\beta)^{K}(J \sqrt{\beta})^{K+1}(1-J \sqrt{\beta})^{-1}, \\
& \sup _{\{x, y\}} \sum_{\substack{y \in T \subset \mathbb{Z}^{d} \\
|T|=K}} \int W_{T}\left(d S_{T}\right)\left|\zeta\left(S_{x} S_{T}\right)\right| \\
& \leqq \leqq \\
& \quad e^{\frac{1}{2} J v_{x}\left(S_{x}\right)^{2}} e^{-\delta(x, y)} I(\beta)^{K}(J \sqrt{\beta})^{K}(1-J \sqrt{\beta})^{-1} .
\end{aligned}
$$

Proof. Our main task is to show (2.7) because the other inequalities follows from obvious modifications of the proof of (2.7). We have

$$
\zeta(R)=\int W_{R}\left(d S_{R}\right) \sum_{g \in C_{R}} \prod_{\{x, y\} \in g}\left(e^{-\beta \Phi_{x y}\left(S_{x} S_{y}\right)}-1\right) .
$$

We use (1.4), the inequality $e^{\lambda t}-1 \leqq t\left(e^{\lambda}-1\right)$ for $0 \leqq t \leqq 1, \lambda \geqq 0$ and (1.9):

$$
\begin{aligned}
& |\zeta(R)| \leqq \sum_{g \in C_{R}} \int_{R} W_{R}\left(d S_{R}\right) \prod_{\{x, y\} \in g}\left(e^{\beta e^{-\delta(x, y)} J(x, y) v_{x}\left(S_{x}\right) v_{y}\left(S_{y}\right)}-1\right) \\
& \leqq \sum_{g \in C_{R}}\left(\prod_{\{x, y\} \in g} \beta e^{-\delta(x, y)} J(x, y)^{2 / 3}\right) \int W_{R}\left(d S_{R}\right) e_{\{x, y\} \in g} J(x, y)^{1 / 3 v_{x}}\left(S_{x}\right) v_{y}\left(S_{y}\right) .
\end{aligned}
$$

The argument of the exponential, for each $g \in C_{R}$, is bounded by

$$
\frac{1}{2} \sum_{\{x, y\} \subset R} v_{x}\left(S_{x}\right)^{2} J(x, y)^{1 / 3}+\frac{1}{2} \sum_{\{x, y\} \subset R} v_{y}\left(S_{y}\right)^{2} J(x, y)^{1 / 3}
$$

and so also by $\frac{1}{2} J \sum_{x \in R} v_{x}\left(S_{x}\right)^{2}$ in force of (1.6).

The integral is so bounded by

$$
\prod_{x \in R} \int W_{x}\left(d S_{x}\right) e^{\frac{1}{2} J v_{x}\left(S_{x}\right)^{2}}
$$


and, using (1.7), we have

$$
|\zeta(R)| \leqq e^{-\delta(R)} I(\beta)^{|R|} \sum_{g \in C_{R}} \prod_{\{x, y\} \in g}\left(\beta J(x, y)^{2 / 3}\right),
$$

where $\delta(R)$ is the smallest length of the graphs in $C_{R}$.

We observe, as in [8], that to each graph $g \in C_{R}$ and to each $x \in R$, one can associate at least one sequence $\left(x_{1}, \ldots, x_{q}\right) \in R^{q}$ with $q \geqq|R|$ such that $x_{1}=x$, $x_{i} \neq x_{i+1},\left\{x_{1}, x_{i+1}\right\} \in g$, and if $\{y, z\} \in g$ there are one or two labels $i$ such that $\left\{x_{i}, x_{i+1}\right\}=\{y, z\}$. This implies, if (1.9) holds,

$$
\begin{aligned}
\sum_{\substack{x \in R \in \mathscr{R} \\
|R|=K}} \sum_{g \in C_{R}} \prod_{\{x, y\} \in g}\left(\beta J(x, y)^{2 / 3}\right) & \leqq \sum_{q=K}^{\infty} \sum_{\substack{\left(x_{1}, \ldots, x_{q}\right) \in\left(\mathbb{Z}^{d}\right) q \\
x_{1}=x, x_{i} \neq x_{i+1}}} \prod_{i=1}^{q-1}\left(\beta^{1 / 2} J\left(x_{i}, x_{i+1}\right)^{1 / 3}\right) \\
& \leqq \sum_{q=K}^{\infty}\left(\beta^{1 / 2} J\right)^{q-1}
\end{aligned}
$$

and (2.7) follows remembering (1.8).

Lemma 2. In the hypothesis of Lemma 1 and (1.10) there is a function $I_{3}(\beta)=O(\sqrt{\beta}), \beta \rightarrow 0$, such that

$$
\left|\frac{\bar{\varrho}_{\Lambda}(X \cup Y)}{\bar{\varrho}_{\Lambda}(X) \bar{\varrho}_{\Lambda}(Y)}-1\right| \leqq \exp \left(I_{3}(\beta) \sum_{x \in X} \sum_{y \in Y} e^{-\delta(x, y)}\right)-1,
$$

and, in particular,

$$
\left|\frac{\bar{\varrho}_{\Lambda}(X \cup Y)}{\bar{\varrho}_{\Lambda}(X) \bar{\varrho}_{\Lambda}(Y)}-1\right| \leqq \exp \left(I_{3}(\beta) D \min \{|X|,|Y|\}\right) e^{-\frac{1}{2} \delta(X, Y)},
$$

where $\delta(X, Y)=\min _{\substack{x \in X \\ y \in Y}} \delta(x, y)$

Proof. The bound (2.7) of Lemma 1 and (1.10) allows us to apply the exponentiation formula (1.21) if we choose

$$
\varepsilon=I_{1}(\beta)=I(\beta) J^{1 / 2} \beta^{1 / 4}(1-J \sqrt{\beta})^{-1}
$$

and we get

$$
\bar{\varrho}_{\Lambda}(X)=\exp -\sum_{n=1}^{\infty} \frac{1}{n !} \sum_{\substack{\left(R_{1}, \ldots, R_{n}\right) \in \mathscr{R}^{n} \\ R_{1} \subset A \\ \exists R_{1} \cap X \neq \emptyset}} \varphi^{T}\left(R_{1}, \ldots, R_{n}\right) \zeta\left(R_{1}\right) \ldots \zeta\left(R_{n}\right) .
$$

It follows, then,

$$
\frac{\bar{\varrho}_{\Lambda}(X \cap Y)}{\bar{\varrho}_{\Lambda}(X) \bar{\varrho}_{\Lambda}(Y)}=\exp \sum_{n=1}^{\infty} \frac{1}{n !} \sum_{\substack{\left(R_{1}, \ldots, R_{n}\right) \in \mathscr{R}^{n} \\ R_{i} \subset A \\ \exists i: R_{\imath} \cap X \neq \emptyset, R_{\imath} \cap Y \neq \emptyset}} \varphi^{T}\left(R_{1}, \ldots, R_{n}\right) \zeta\left(R_{1}\right) \ldots \zeta\left(R_{n}\right) .
$$


The argument of the exponential is bounded by

$$
\begin{aligned}
& \sum_{n=1}^{\infty} \frac{1}{n !} \sum_{\substack{R \in \mathscr{R} \\
R \cap X \neq \emptyset, \boldsymbol{R} \cap Y \neq \emptyset}} \sum_{\substack{\left(R_{1}, \ldots, R_{n}\right) \in \mathscr{R}^{n} \\
\exists i, R_{1}=R}}\left|\varphi^{T}\left(R_{1}, \ldots, R_{n}\right) \zeta\left(R_{1}\right) \ldots \zeta\left(R_{n}\right)\right| \\
& \leqq \sum_{\substack{x \in X \\
y \in Y}} \sum_{\{x, y\} \subset \in R \in \mathscr{R}} \sum_{n=1}^{\infty} \frac{1}{n !} \sum_{\substack{\left(R_{1}, \ldots, R_{n}\right) \in \mathscr{R}^{n} \\
\exists i: R_{l}=R}}\left|\varphi^{T}\left(R_{1}, \ldots, R_{n}\right) \zeta\left(R_{1}\right) \ldots \zeta\left(R_{n}\right)\right| \\
& \leqq \sum_{\substack{x \in X \\
y \in Y}} \sum_{\{x, y\} \subset R \in \mathscr{R}}|\zeta(R)|\left(1+|R| e^{|R| \frac{1}{2}} \ln \left(1-2 e \frac{I_{1}(\beta)}{1-I_{1}(\beta)}\right)^{-1}\right) \\
& =\sum_{\substack{x \in X \\
y \in Y}} \sum_{K=2}^{\infty} \sum_{\substack{\{x, y\} \subset R \in \mathscr{R} \\
|R|=K}}|\zeta(R)|\left(1+K e^{K} I_{2}(\beta)\right),
\end{aligned}
$$

where we have used (1.20) and we have put

$$
I_{2}(\beta)=\frac{1}{2} \ln \left(1-2 e \frac{I_{1}(\beta)}{1-I_{1}(\beta)}\right)^{-1} .
$$

The last expression can be bounded, using (2.8), by

$$
\begin{aligned}
& \sum_{\substack{x \in X \\
y \in Y}} \sum_{K=2}^{\infty}\left(1+K e^{K} I_{2}(\beta)\right) e^{-\delta(x, y)} I(\beta)^{K}(J \sqrt{\beta})^{K-1}(1-J \sqrt{\beta})^{-1} \\
& \quad=\sum_{x \in X} \sum_{y \in Y} e^{-\delta(x, y)} I_{3}(\beta)
\end{aligned}
$$

where $I_{3}(\beta)$ is defined by the last equation, the series converges by (1.8) and this proves the lemma.

In order to prove Theorem 1 we need to estimate each one of the terms $\Sigma_{1}, \ldots, \Sigma_{6}$. We have by $(2.9)$

$$
\begin{aligned}
\left|\Sigma_{1}\right| & \leqq \sum_{T \subset A \backslash\{x, y\}}\left|\zeta\left(S_{x} S_{y} T\right)\right| \\
& \leqq \sum_{K=0}^{\infty} e^{\frac{1}{2} J v_{x}\left(S_{x}\right)^{2}+\frac{1}{2} J v_{y}\left(S_{y}\right)^{2}} e^{-\delta(x, y)} \frac{I(\beta)^{K}(J \sqrt{\beta})^{K+1}}{1-J \sqrt{\beta}} \\
& =e^{\frac{1}{2} J v_{x}\left(S_{x}\right)^{2}+\frac{1}{2} J v_{y}\left(S_{y}\right)^{2}} e^{-\delta(x, y)} I_{4}(\beta)
\end{aligned}
$$

where $I_{4}(\beta)$ is defined by the last equation (in the same way are defined all the functions $I$ in the following).

We have in the second term from Lemma 2

$$
\left|\varrho_{\Lambda}\left(x y T_{1} T_{2}\right)-\bar{\varrho}_{\Lambda}\left(x T_{1}\right) \bar{\varrho}_{\Lambda}\left(y T_{2}\right)\right| \leqq e^{-\frac{1}{2} \delta\left(x T_{1}, y T_{2}\right)} \exp D I_{3}(\beta)\left(\left|T_{1}\right|+1\right) .
$$

For the term in $\Sigma_{2}$ with $T_{1} \neq \emptyset$ and $T_{2} \neq \emptyset$ we use the bound

$\sum_{\substack{t_{1}, t_{2} \in \Lambda \backslash\{x, y\} \\ t_{1} \neq t_{2}}} \sum_{\substack{t_{1} \in T_{1} \subset \Lambda \backslash\{x, y\} \\ \delta\left(x, T_{1}\right) \leqq \delta\left(x, t_{1}\right)}} \sum_{\substack{t_{2} \in T_{2} \subset A \backslash\left\{\left(y, T_{2}\right) \leqq \delta, y\right\} \backslash T_{1} \\ \delta\left(y, t_{2}\right)}}\left|\zeta\left(S_{x} T_{1}\right) \zeta\left(S_{y} T_{2}\right)\right| e^{-\frac{1}{2} \delta\left(x T_{1}, y T_{2}\right)} e^{D I_{3}(\beta)\left(\left|T_{1}\right|+1\right)}$ 


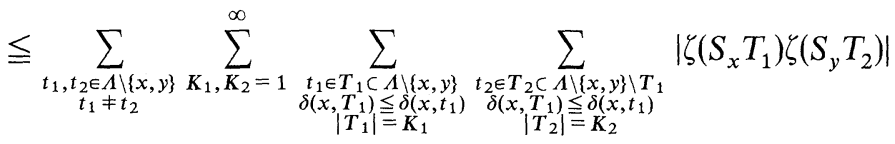

$$
\begin{aligned}
& \cdot e^{D I_{3}(\beta)\left(\left|T_{1}\right|+1\right)} e^{-\frac{1}{2} \delta(x, y)+\frac{1}{2} \delta\left(x, t_{1}\right)+\frac{1}{2} \delta\left(y, t_{2}\right)} \\
& \leqq e^{-\frac{1}{2} \delta(x, y)} \sum_{t_{1} \in \Lambda \backslash\{x, y\}} e^{-\frac{1}{2} \delta\left(x, t_{1}\right)} \sum_{t_{2} \in \Lambda \backslash\left\{x, y, t_{1}\right\}} e^{-\frac{1}{2} \delta\left(y, t_{2}\right)} \sum_{K_{1}=1}^{\infty} e^{D I_{3}(\beta)\left(K_{1}+1\right)} e^{\frac{1}{2} J v_{x}\left(S_{x}\right)^{2}} \\
& \cdot I(\beta)^{K_{1}}(J \sqrt{\beta})^{K_{1}}(1-J \sqrt{\beta})^{-1} \sum_{K_{2}=1}^{\infty} e^{\frac{1}{2} J v_{y}\left(S_{y}\right)^{2}} I(\beta)^{K_{2}}(J \sqrt{\beta})^{K_{2}}(1-J \sqrt{\beta})^{-1} \\
& \leqq e^{-\frac{1}{2} \delta(x, y)} e^{\frac{1}{2} J v_{x}\left(S_{x}\right)^{2}+\frac{1}{2} J v_{y}\left(S_{y}\right)^{2}} I_{5}(\beta),
\end{aligned}
$$

where we have used (1.8) and $\delta(x, y) \leqq \delta\left(x, t_{1}\right)+\delta\left(y, t_{2}\right)+\delta\left(x T_{1}, y T_{2}\right)$. The terms with $T_{1}=\emptyset$ or $T_{2}=\emptyset$ must be separately estimated. For $T_{1}=\emptyset$ and $T_{2} \neq \emptyset$ we have

$$
\begin{aligned}
& \sum_{t_{2} \in \Lambda \backslash\{x, y\}} \sum_{t_{2} \in T_{2} \subset \Lambda \backslash\{x, y\}}\left|\zeta\left(S_{y} T_{2}\right)\right| e^{-\frac{1}{2} \delta\left(x, y T_{2}\right)} e^{D I_{3}(\beta)} \\
& \leqq \sum_{\left.t_{2} \in \Lambda \backslash\{x, y\}\right\}} \sum_{K_{2}=1}^{\infty} \sum_{\substack{t_{2} \in T_{2} \subset A \backslash\{x, y\} \\
\left|T_{2}\right|=K 2 \\
\delta\left(y, T_{2}\right) \leqq \delta\left(y, t_{2}\right)}}\left|\zeta\left(S_{y} T_{2}\right)\right| e^{-\frac{1}{2} \delta(x, y)+\frac{1}{2} \delta\left(y, t_{2}\right)} e^{D I_{3}(\beta)} \\
& \leqq e^{-\frac{1}{2} \delta(x, y)} \sum_{t_{2} \in \Lambda \backslash\{x, y\}} e^{-\frac{1}{2} \delta\left(y, t_{2}\right)} \sum_{K_{2}=1}^{\infty} e^{-\frac{1}{2} J v_{y}\left(S_{y}\right)^{2}} \\
& \cdot I(\beta)^{K_{2}}(J \sqrt{\beta})^{K_{2}}(1-J \sqrt{\beta})^{-1} e^{D I_{3}(\beta)} \\
& \leqq e^{-\frac{1}{2} \delta(x, y)} e^{\frac{1}{2} J v_{y}\left(S_{y}\right)^{2}} I_{6}(\beta),
\end{aligned}
$$

where we have used $\delta(x, y) \leqq \delta\left(x, y T_{2}\right)+\delta\left(y, t_{2}\right)$. The term with $T_{1} \neq \emptyset$ and $T_{2}=\emptyset$ give the same contribution, while the term with $T_{1}=T_{2}=\emptyset$ gives a contribution less than

$$
\left(e^{I_{3}(\beta)}-1\right) e^{-\delta(x, y)}
$$

We so get

$$
\left|\Sigma_{2}\right| \leqq e^{-\frac{1}{2} \delta(x, y)} e^{\frac{1}{2} J v_{x}\left(S_{x}\right)^{2}+\frac{1}{2} J v_{y}\left(S_{y}\right)^{2}} I_{7}(\beta) .
$$

The third term in (2.6) is bounded by

$$
\begin{aligned}
& \sum_{t \in \Lambda \backslash\{x, y\}} \sum_{K_{1}, K_{2}=1}^{\infty} \sum_{\substack{t \in T_{1} C \Lambda \Lambda\{x, y\} \\
\left|T_{1}\right|=K_{1}}} \sum_{\substack{t \in T_{2} C \Lambda \Lambda\{x, y\} \\
\left|T_{2}\right|=K_{2}}}\left|\zeta\left(S_{x} T_{1}\right) \zeta\left(S_{y} T_{2}\right)\right| \\
& \leqq \sum_{t \in \Lambda \backslash\{x, y\}} \sum_{K_{1}, K_{2}=1}^{\infty} e^{-\delta(x, t)-\delta(y, t)} e^{\frac{1}{2} J v_{x}\left(S_{x}\right)^{2}+\frac{1}{2} J v_{y}\left(S_{y}\right)^{2}} \frac{I(\beta)^{K_{1}+K_{2}}(J \sqrt{\beta})^{K_{1}+K_{2}}}{(1-J \sqrt{\beta})^{2}} \\
& \leqq e^{-\frac{1}{2} \delta(x, y)} e^{\frac{1}{2} J v_{x}\left(S_{x}\right)^{2}+\frac{1}{2} J v_{y}\left(S_{y}\right)^{2}} I_{8}(\beta) .
\end{aligned}
$$

The fourth term in (2.6) is bounded by

$$
\sum_{K_{1}=1}^{\infty} \sum_{\substack{y \in T_{1} \subset \Lambda \backslash\{x\} \\\left|T_{1}\right|=K_{1}}}\left|\zeta\left(\mathrm{S}_{x} \mathrm{~T}_{1}\right)\right| \sum_{K_{2}=0}^{\infty} \sum_{\substack{t_{2} \in \Lambda \backslash\{x, y\} \\ t_{2} \in T_{2} \subset \Lambda \Lambda\{x, y\} \\\left|T_{2}\right|=K_{2}}}\left|\zeta\left(\mathrm{S}_{y} \mathrm{~T}_{2}\right)\right|
$$




$$
\begin{aligned}
& \leqq \sum_{K_{1}=1}^{\infty} e^{-\delta(x, y)} e^{\frac{1}{2} J v_{x}\left(S_{x}\right)^{2}} I(\beta)^{K_{1}}(J \sqrt{\beta})^{K_{1}}(1-J \sqrt{\beta})^{-1} \\
& \cdot \sum_{K_{2}=0}^{\infty} \sum_{t_{2} \in \Lambda \backslash\{x, y\}} e^{-\delta\left(y, t_{2}\right)} e^{\frac{1}{2} J v_{y}\left(S_{y}\right)^{2}} I(\beta)^{K_{2}}(J \sqrt{\beta})^{K_{2}}(1-J \sqrt{\beta})^{-1} \\
& \leqq e^{-\delta(x, y)} e^{\frac{1}{2} J v_{x}\left(S_{x}\right)+\frac{1}{2} J v_{y}\left(S_{y}\right)^{2}} I_{9}(\beta) .
\end{aligned}
$$

The fifth term gives the same contribution as the fourth and the sixth gives

$$
\begin{aligned}
& \sum_{K_{1}=1}^{\infty} \sum_{\substack{y \in T_{1} \subset \Lambda|\{x\}\\
| T_{1} \mid=K_{1}}}\left|\zeta\left(S_{x} T_{1}\right)\right| \sum_{K_{2}=1}^{\infty} \sum_{\substack{x \in T_{2} \subset A \Lambda\{y\} \\
\left|T_{2}\right|=K_{2}}}\left|\zeta\left(S_{y} T_{2}\right)\right| \\
& \leqq e^{-2 \delta(x, y)} e^{\frac{1}{2} J v_{x}\left(S_{x}\right)^{2}+\frac{1}{2} J v_{y}\left(S_{y}\right)^{2}} I_{10}(\beta) .
\end{aligned}
$$

Collecting the six bounds we finally get (1.11) and it is easy to see that

$$
I_{11}(\beta)=O(\sqrt{\beta}), \quad \beta \rightarrow 0 .
$$

\section{Proof of Theorem 2}

We rewrite Eq. (1.13) in the form

$$
\begin{aligned}
Z_{\Lambda} & =1+\sum_{n \geqq 1} \frac{1}{n !} \sum_{\substack{\left(R_{1}, \ldots, R_{n}\right) \in \mathscr{R}^{n} \\
R_{i} \cap R_{j}=\emptyset, R_{i} \subset A}} \zeta\left(\mathrm{R}_{1}\right) \ldots \zeta\left(\mathrm{R}_{n}\right) \\
& =1+\sum_{n \geqq 1} \frac{1}{n !} \sum_{\substack{\left.R_{1}, \ldots, R_{n}\right) \in \mathscr{R}^{n} \\
\boldsymbol{R}_{i} \subset A}} \zeta\left(R_{1}\right) \ldots \zeta\left(R_{n}\right) \prod_{\substack{i, j\} \subset\{1, \ldots, n\}\\
}} \chi\left(R_{i}, R_{j}\right),
\end{aligned}
$$

and we insert in the last expression the expansion

$$
\prod_{\{i, j\} \subset\{1, \ldots, n\}} \chi\left(R_{i}, R_{j}\right)=\sum_{K=1}^{n} \sum_{\left\{I_{1}, \ldots, I_{K}\right\} \in \pi(\{1, \ldots, n\})} \varphi^{T}\left(R_{h}, h \in I_{1}\right) \ldots \varphi^{T}\left(R_{h}, h \in I_{K}\right) .
$$

So we get, at least formally, the exponentiation formula (1.21) exchanging the order of summation. This exchange can be done if the series

$$
\sum_{n=1}^{\infty} \frac{1}{n !} \sum_{\substack{\left(R_{1}, \ldots, R_{n}\right) \in \mathscr{R}^{n} \\ \boldsymbol{R}_{i} \subset \Lambda}} \varphi^{T}\left(\mathrm{R}_{1}, \ldots, \mathrm{R}_{n}\right) \zeta\left(\mathrm{R}_{1}\right) \ldots \zeta\left(\mathrm{R}_{n}\right)
$$

is absolutely convergent. This follows from (1.20) if we use

$$
\sum_{\substack{\left(R_{1}, \ldots, R_{n}\right) \in \mathscr{R}^{n} \\ R_{i} \subset A}}(\ldots) \leqq \sum_{\substack{R \in \mathscr{R} \\ R \subset A}} \sum_{\substack{\left.R_{1}, \ldots, R_{n}\right) \in \mathscr{R}^{n} \\ \exists R_{i}=R}}(\ldots)
$$

and the bound (1.18). In order to prove (1.20) we observe that

$$
\sum_{n=1}^{\infty} \frac{1}{n !} \sum_{\substack{\left(R_{1}, \ldots, R_{n}\right) \in \mathscr{R} \mathfrak{R}^{n} \\ \exists \in R_{1}=R}}\left|\varphi^{T}\left(\mathrm{R}_{1}, \ldots, \mathrm{R}_{n}\right) \zeta\left(\mathrm{R}_{1}\right) \ldots \zeta\left(\mathrm{R}_{n}\right)\right|
$$




$$
\begin{aligned}
& \left.\leqq|\zeta(R)|\left(1+\sum_{n=2}^{\infty} \frac{1}{n !} \sum_{i=1}^{n} \sum_{\substack{\left(R_{1}, \ldots, R_{n}\right) \in \mathscr{R}^{n} \\
R_{i}=R}} \mid \varphi^{T} \mathrm{R}_{1}, \ldots, \mathrm{R}_{n}\right) \prod_{K \neq i} \zeta\left(\mathrm{R}_{K}\right) \mid\right) \\
& \leqq \zeta \zeta(R) \mid\left(1+\sum_{n=2}^{\infty} \frac{n}{n !} \sum_{\left(R_{2}, \ldots, R_{n}\right) \in \mathscr{R}^{n-1}}\left|\varphi^{T}\left(\mathrm{R}_{1}, \ldots, \mathrm{R}_{n}\right) \zeta\left(\mathrm{R}_{2}\right) \ldots \zeta\left(\mathrm{R}_{n}\right)\right|\right),
\end{aligned}
$$

and so our task is reduced to estimate the sum

$$
\sum_{\left(R_{2}, \ldots, R_{n}\right) \in \mathscr{R ^ { n } - 1}}\left|\varphi^{T}\left(R_{1}, \ldots, R_{n}\right) \zeta\left(R_{2}\right) \ldots \zeta\left(R_{n}\right)\right| .
$$

We rewrite this sum as a sum over the connected graphs on $\{1, \ldots, n\}$ using that $\varphi^{T}\left(R_{1}, \ldots, R_{n}\right)$ depends only on the graph $g\left(R_{1}, \ldots, R_{n}\right)$. If we define the function $\varphi$ on $C_{n}$ by

$$
\varphi(g)= \begin{cases}\sum_{\substack{f \subset g \\ f \in C_{n}}}^{1}(-1)^{|f|} & n=1 \\ & n>1\end{cases}
$$

we have

$$
\varphi^{T}\left(R_{1}, \ldots, R_{n}\right)=\varphi\left(g\left(R_{1}, \ldots, R_{n}\right)\right),
$$

and so (3.6) is equal to

$$
\sum_{f \in C_{n}}|\varphi(f)| \sum_{\substack{\left(R_{2}, \ldots, R_{n}\right) \in \mathscr{R}^{n-1} \\ g\left(\boldsymbol{R}_{1}, \ldots, R_{n}\right)=f}}\left|\zeta\left(R_{1}\right) \ldots \zeta\left(R_{n}\right)\right| .
$$

We use the following nontrivial bound for $\varphi(f)$ in terms of $N(f)$, the number of trees contained in $f$.

\section{Proposition}

$$
|\varphi(f)| \leqq N(f) .
$$

For the proof we refer to $[10]$ or to $[13,14]$. From

$$
\sum_{f \in C_{n}}(\ldots)=\sum_{t \in T_{n}} \sum_{f \supset t} \frac{1}{N(f)}(\ldots),
$$

where $T_{n}$ is the set of the trees on $\{1, \ldots, n\}$, we get

$$
\begin{aligned}
(3.8) & \leqq \sum_{t \in T_{n}} \sum_{f \supset t} \sum_{\substack{\left(R_{2}, \ldots, R_{n}\right) \in \mathscr{R} \text { n-1 } \\
g\left(R_{1}, \ldots, R_{n}\right)=f}}\left|\zeta\left(R_{2}\right) \ldots \zeta\left(R_{n}\right)\right| \\
& =\sum_{t \in T_{n}} \sum_{\substack{\left(R_{2}, \ldots, R_{n}\right) \in \mathscr{R}^{n-1} \\
g\left(R_{1}, \ldots, R_{n}\right) \supset t}}\left|\zeta\left(R_{2}\right) \ldots \zeta\left(R_{n}\right)\right| \\
& =\sum_{t \in T_{n}} w(t)
\end{aligned}
$$

where the definition of $w(t)$ is implicit in the last equation. Let us compute $w(t)$, for instance, for the tree on $\{1,2,3,4\}$ made by the lines $\{1,2\},\{2,3\},\{2,4\}$. We 
have

$$
w(t)=\sum_{\substack{R_{2} \in \mathscr{R} \\ R_{2} \cap R_{1} \neq \emptyset}}\left|\zeta\left(R_{2}\right)\right| \sum_{\substack{R_{3} \in \mathscr{R} \\ R_{3} \cap R_{2} \neq \emptyset}}\left|\zeta\left(R_{3}\right)\right| \sum_{\substack{R_{4} \in \mathscr{R} \\ R_{4} \cap R_{2} \neq \emptyset}}\left|\zeta\left(R_{4}\right)\right| .
$$

The sum over $R_{4}$ gives by (1.18) a contribution less than

$$
\varepsilon(1-\varepsilon)^{-1}\left|R_{2}\right|
$$

and the same does the sum over $R_{3}$. We so have

$$
w(t) \leqq\left(\frac{\varepsilon}{1-\varepsilon}\right)^{2}\left|R_{1}\right| \sup _{x} \sum_{x \in R_{2} \in \mathscr{R}}\left|\zeta\left(R_{2}\right)\right|\left|R_{2}\right|^{2} .
$$

We are so led to estimate the series

$$
\sum_{x \in R \in \mathscr{R}}|\zeta(R)||R|^{p}
$$

for each nonnegative integer $p$ and this can be done using, for instance, the bound

$$
\sum_{K=1}^{\infty} \varepsilon^{K} K^{p} \leqq p ! \frac{\varepsilon}{1-\varepsilon}
$$

that holds if $\varepsilon(1-\varepsilon)^{-1}<(e-1)^{-1}$ and follows from a simple induction argument. We so find

$$
w(t) \leqq\left(\frac{\varepsilon}{1-\varepsilon}\right)^{3}\left|R_{1}\right| 2 !
$$

Generally, for a tree $t$ such that the degree, i.e. the number of lines containing the point $i \in\{1, \ldots, n\}$ is $d_{i}$, we have

$$
w(t) \leqq\left|R_{1}\right|^{d_{1}}\left(\frac{\varepsilon}{1-\varepsilon}\right)^{n-1} \prod_{i=2}^{n}\left(d_{i}-1\right) !
$$

The number of trees on $\{1, \ldots, n\}$ such that the degree of the point $i$ is $d_{i}$ is given, by the Cayley formula [12], by

$$
\frac{(n-2) !}{\prod_{i=1}^{n}\left(d_{i}-1\right) !}
$$

The sum over the trees can be performed summing over the sequences $\left(d_{1}, \ldots, d_{n}\right) \in I_{n-1}^{n}$, where $I_{n}=\{1, \ldots, n\}$, with the constraint $d_{1}+\ldots+d_{n}=2(n-1)$. (3.9) is so bounded by

$$
\begin{aligned}
& \sum_{\substack{\left(d_{1}, \ldots, d_{n}\right) \in I_{n-1}^{n}-1 \\
d_{1}+\ldots+d_{n}=2(n-1)}} \frac{(n-2) !}{\prod_{i=1}^{n}\left(d_{i}-1\right) !}\left|R_{1}\right|^{d_{1}}\left(\frac{\varepsilon}{1-\varepsilon}\right)^{n-1} \prod_{i=2}^{n}\left(d_{i}-1\right) ! \\
= & \left(\frac{\varepsilon}{1-\varepsilon}\right)^{n-1}(n-2) ! \sum_{d_{1}=1}^{n-1} \frac{\left|R_{1}\right|^{d_{1}}}{\left(d_{1}-1\right) !} \sum_{\substack{\left(d_{2}, \ldots, d_{n}\right) \in I_{n}^{n-1} \\
d_{2}+\ldots+d_{n}=2(n-1)-d_{1}}} 1 .
\end{aligned}
$$

We now need a bound of the sum over $\left(d_{2}, \ldots, d_{n}\right) \in I_{n-1}^{n-1}$. This sum can be bounded, for instance, with the sum over $\left(d_{2}, \ldots, d_{n}\right) \in I_{2(n-1)-d_{1}}^{n-1}$ that we can denote 
$\Gamma_{n-1}\left(2(n-1)-d_{1}\right)$ if we define for $1 \leqq K \leqq m$

$$
\Gamma_{K}(m)=\sum_{\substack{\left(q_{1}, \ldots, q_{K}\right) \in I_{m}^{K} \\ q_{1}+\ldots+q_{K}=m}} 1 .
$$

But we have, via a simple induction argument on $K$

and so

$$
\Gamma_{K}(m) \leqq \frac{m^{K-1}}{(K-1) !}
$$

$$
\Gamma_{n-1}\left(2(n-1)-d_{1}\right) \leqq \frac{\left(2(n-1)-d_{1}\right)^{n-2}}{(n-2) !} \leqq \frac{1}{2}(2 e)^{n-1} .
$$

Finally (3.6) is less than

$$
\frac{1}{2}\left(2 e \frac{\varepsilon}{1-\varepsilon}\right)^{n-1}(n-2) !\left|R_{1}\right| e^{\left|R_{1}\right|}
$$

and Eq. (1.20) follows summing the series (3.5).

Acknowledgement. We are grateful for many discussions with E. Olivieri, M. Cassandro, and D. Capocaccia.

\section{References}

1. Kunz, H.: Analiticity and clustering properties of unbounded spin systems. Commun. Math. Phys. 59, 53-69 (1978)

2. Israel, R.B., Nappi, C.R.: Exponential clustering for long-range integer spin systems. Commun. Math. Phys. 68, 29-37 (1979)

3. Gross, L.: Decay of correlations in classical lattice models at high temperature. Commun. Math. Phys. 68, 9-27 (1979)

4. Duneau, M., Souillard, B.: Cluster properties of lattice and continuous systems. Commun. Math. Phys. 47, 155-166 (1976)

5. Gruber, C., Kunz, H.: General properties of polymer systems. Commun. Math. Phys. 22, 133-161 (1971)

6. Gallavotti, G., Martin-Löf, A., Miracle-Solé, S. : Some problems connected with the description of cohexisting phases at low temperature in the Ising model. In : Lecture Notes in Physics, Vol. 20, pp. 162-202. Berlin, Heidelberg, New York: Springer 1971

7. Ruelle, D. : Statistical mechanics. New York: Benjamin 1969

8. Campanino, M., Capocaccia, D., Tirozzi, B.: The local central limit theorem for a Gibbs random field. Commun. Math. Phys. 70, 125-132 (1979)

9. Cassandro, M., Olivieri, E.: Renormalization group and analiticity in one dimension: A proof of the Dobrushin's Theorem. Commun. Math. Phys. 80, 255-269 (1981)

10. Penrose, O.: Convergence of fugacity expansions for classical systems. In: Statistical mechanics : foundations and applications, Bak, A. (ed.). New York: Benjamin 1967

11. Brydges, D., Federbush, P.: A new form of the Mayer expansion in classical statistical mechanics. J. Math. Phys. 19, 2064-2067 (1978)

12. Moon, J.W.: Enumerating labelled trees. In: Graph theory and theoretical physics, Harary, F. (ed.). New York: Academic Press 1967

13. Rota, G.C.: Z. Wahrscheinlichkeitstheorie Verw. Gebiete 2, 340 (1964)

14. Malyshev, V.A.: Uniform cluster estimates for lattice models. Commun. Math. Phys. 64, 131-157 (1979)

Communicated by E. Lieb

Received December 10, 1981 